\title{
ON THE NUMBER OF SIGN CHANGES OF HECKE EIGENVALUES OF NEWFORMS
}

\author{
WINFRIED KOHNEN, YUK-KAM LAU and IGOR E. SHPARLINSKI ${ }^{凶}$
}

(Received 19 April 2006; accepted 29 June 2006)

Communicated by W. W. L. Chen

\begin{abstract}
We show that, for every $x$ exceeding some explicit bound depending only on $k$ and $N$, there are at least $C(k, N) x / \log ^{17} x$ positive and negative coefficients $a(n)$ with $n \leq x$ in the Fourier expansion of any non-zero cuspidal Hecke eigenform of even integral weight $k \geq 2$ and squarefree level $N$ that is a newform, where $C(k, N)$ depends only on $k$ and $N$. From this we deduce the existence of a sign change in a short interval.
\end{abstract}

2000 Mathematics subject classification: 11F11,11F30.

Keywords and phrases: Hecke eigenvalues, sign changes.

\section{Introduction}

Let $f$ be a non-zero cusp form of even integral weight $k \geq 2$ and level $N$ with real Fourier coefficients $a(n), n \in \mathbb{N}$. We refer to [11] for basic definitions. It is well known that there are infinitely many $n \in \mathbb{N}$ such that $a(n)>0$ as well as infinitely many $n$ with $a(n)<0$. For an extension of this result and a discussion of related questions, see [8] (compare also [2] in connection with binary theta functions).

If $N=1$ and $k \equiv 2(\bmod 4)$, then a result of Siegel [12] implies that the first sign change of $a(n)$ already occurs among the first $d(k)+1$ coefficients, where $d(k)$ is the dimension of the space of cusp forms in question (see also [3]). On the other hand, if $N=1$ and $k \equiv 0(\bmod 4)$ or if $N>1$, the method of Siegel [12] does not apply and thus a different approach, based on analytic number theory estimates, has been developed by Kohnen and Sengupta [9], which in turn is related to some ideas of Murty [10].

More precisely, let $f$ be a fixed newform of weight $k$ on the Hecke congruence subgroup

$$
\Gamma_{0}(N)=\left\{\left(\begin{array}{ll}
a & b \\
c & d
\end{array}\right) \in S L_{2}(\mathbf{Z}) \mid c \equiv 0 \quad(\bmod N)\right\},
$$

The third author was supported in part by ARC grant DP0556431 during the preparation of this paper.

(C) 2008 Australian Mathematical Society 1446-7887/08 \$A2.00+0.00 
which is a normalized Hecke eigenform. In particular, its Fourier coefficients $a(n)$, $n \in \mathbb{N}$, are the Hecke eigenvalues of $f$ and $a(1)=1$. Note that the $a(n)$ are real.

We assume throughout that $N$ is squarefree.

As in [9], we note that it is quite reasonable to assume that $\operatorname{gcd}(n, N)=1$ since the $p$-eigenvalues of $f$ for $p \mid N$ are explicitly known.

In the following the implied constants in the symbols $\ll$ are always absolute and efficiently computable.

It is shown in [9] that for any $\varepsilon>0$ there exist $n \in \mathbb{N}$ with $\operatorname{gcd}(n, N)=1$ and such that

$$
n \ll k N \exp \left(c \sqrt{\frac{\log N}{\log \log (N+2)}}\right) \log ^{26+\varepsilon} k,
$$

for which $a(n)<0$, where $c$ is an absolute constant and the implied constant depends only on $\varepsilon$. This bound has recently been improved by Iwaniec, Kohnen and Sengupta [7].

Here we show that the technique of [9] can in fact give a lower bound on the number of sign changes in a given interval $n \in[1, x]$. On the other hand, the approach of [7], which led to an improvement of (1), does not seem to apply immediately to the derivation of a lower bound on the number of sign changes.

To formulate our result, we introduce the divisor sums

$$
\sigma_{\alpha}(N)=\sum_{d \mid N} d^{\alpha}
$$

Let $S_{f}^{+}(x)$ and $S_{f}^{-}(x)$ denote the number of positive integers $n \leq x$ with $\operatorname{gcd}(n, N)=1$ for which $a(n)>0$ and $a(n)<0$, respectively.

THEOREM 1. We have

$$
S_{f}^{ \pm}(x) \gg \frac{x}{\sigma_{-1}(N)^{4} \log ^{4}(k N) \log ^{17} x}
$$

whenever $x \geq X(k, N)$, where

$$
X(k, N)=C k \max \left\{N \sigma_{-1}(N)^{4} \sigma_{-1 / 2}(N)^{2} \log ^{8}(k N), N^{1 / 2} \sigma_{-1}(N)^{6} \log ^{22}(k N)\right\},
$$

for some absolute constant $C>0$.

We also show that Theorem 1, coupled with a recent result of Alkan and Zaharescu [1], allows us to study sign changes in short intervals.

THEOREM 2. There are absolute constants $\eta<1$ and $A>0$ such that, for $y=x^{\eta}$,

$$
S_{f}^{ \pm}(x+y)-S_{f}^{ \pm}(x)>0
$$

whenever $x \geq(k N)^{A}$. 
Let $T_{f}(x)$ denote the number of sign changes in the sequence $a(n)$ taken for consecutive positive integers $n \leq x$ with $\operatorname{gcd}(n, N)=1$, that is,

$$
T_{f}(x)=\#\{n \leq x \mid \operatorname{sign}(a(n)) \neq \operatorname{sign}(a(n+1)), \operatorname{gcd}(n, N)=1\},
$$

where, as usual,

$$
\operatorname{sign}(a)= \begin{cases}-1 & \text { if } a<0 \\ 0 & \text { if } a=0 \\ 1 & \text { if } a>0\end{cases}
$$

Splitting the interval $[1, x]$ into $x^{1-\eta}$ intervals of length $y=x^{\eta}$, we derive from Theorem 2 the following result.

COROLlARY 3. There are absolute constants $\kappa>0$ and $A>0$ such that

$$
T_{f}(x)>x^{\kappa}
$$

whenever $x \geq(k N)^{A}$.

\section{Preparations}

2.1. The idea of the proof We define the 'normalized' Hecke eigenvalues $\lambda(n)$ of $f$ by the relation

$$
a(n)=\lambda(n) n^{(k-1) / 2}, \quad n \in \mathbb{N} .
$$

We now consider the sums

$$
\vartheta_{v}(x)=\sum_{\substack{n \leq x \\ \operatorname{gcd}(n, N)=1}}|\lambda(n)|^{\nu} \log ^{2}(x / n) \quad \text { and } \rho_{\nu}(x)=\sum_{\substack{n \leq x \\ \operatorname{gcd}(n, N)=1}} \lambda(n)^{\nu} \log ^{2}(x / n),
$$

which we use only for $v=1,2,3$.

By the Cauchy-Schwarz inequality,

$$
\vartheta_{2}(x) \leq \sqrt{\vartheta_{1}(x) \vartheta_{3}(x)} .
$$

The proof of Theorem 1 is based on the observation that, if either $S_{f}^{+}(x)$ or $S_{f}^{-}(x)$ is small, then the sums $\vartheta_{1}(x)$ are close to the sum $\left|\rho_{1}(x)\right|$. But the known lower bound on $\vartheta_{2}(x)$ and the known upper bounds on $\rho_{1}(x)$ and $\vartheta_{3}(x)$ contradict (2).

The proof of Theorem 2 is based on the observation that Theorem 1 implies that, for any $\varepsilon>0$ and a sufficiently large $X$, there are $m$ and $n$ with $X \leq m<n \leq X^{1+\varepsilon}$ which are close to each other and also satisfy

$$
\operatorname{gcd}(m n, N)=1, \quad \lambda(m) \lambda(n)<0 .
$$

After this selection of $s$ with $\operatorname{gcd}(s, m n N)=1$ in an appropriate interval (depending on $m$ and $n)$ and such that $\lambda(s) \neq 0$, the existence of which is implied by a result of [1], we can make sure that both $s m$ and $s n$ belong to the desired short interval and we also have

$$
\lambda(s m) \lambda(s n)=\lambda(s)^{2} \lambda(m) \lambda(n)<0 .
$$


2.2. Some elementary bounds We need some elementary number theoretic estimates.

Recalling that $N$ is squarefree we immediately obtain the following results.

LEMMA 4. We have

$$
\prod_{p \mid N}\left(1+p^{-1}\right)=\sigma_{-1}(N)
$$

LEMMA 5. We have

$$
\prod_{p \mid N}\left(1-p^{-1 / 2}\right) \gg \frac{1}{\sigma_{-1}(N) \sigma_{-1 / 2}(N)}
$$

PROOF. Using the identity

$$
\begin{aligned}
\prod_{p \mid N}\left(1-p^{-1 / 2}\right) & =\prod_{p \mid N}\left(1-p^{-1}\right) \prod_{p \mid N}\left(1+p^{-1 / 2}\right)^{-1} \\
& =\prod_{p \mid N}\left(1-p^{-1}\right) \sigma_{-1 / 2}(N)^{-1} \\
& =\prod_{p \mid N}\left(1-p^{-2}\right) \prod_{p \mid N}\left(1+p^{-1}\right)^{-1} \sigma_{-1 / 2}(N)^{-1} \\
& =\prod_{p \mid N}\left(1-p^{-2}\right) \sigma_{-1}(N)^{-1} \sigma_{-1 / 2}(N)^{-1}
\end{aligned}
$$

yields the desired result.

Let $\tau(n)=\sigma_{0}(n)$ be the number of positive integer divisors of $n$. We need the following well-known bounds (see [4, 6]).

LEMMA 6. For any $z \geq 1$, we have

$$
\sum_{n \leq z} \tau(n)^{2} \ll z \log ^{3} z \text { and } \sum_{n \leq z} \tau(n)^{3} \ll z \log ^{7} z .
$$

2.3. Some bounds for sums $\vartheta_{v}(x)$ and $\rho_{v}(x)$ The following estimate is a combination of [9, Proposition 6] with a result of Goldfield, Hoffstein and Lieman [5] (which has also been used in [9]) as well as Lemmas 4 and 5.

LEMMA 7. There are absolute constants $c_{1}, c_{2}>0$ such that the bound

$$
\vartheta_{2}(x) \geq \frac{c_{1}}{\sigma_{-1}(N) \log (k N)} x-c_{2}(k N)^{1 / 2} \log ^{3}(k N) \sigma_{-1}(N) \sigma_{-1 / 2}(N) x^{1 / 2}
$$

holds for every $x \geq 1$.

Using Lemma 4 instead of [9, Lemma 4] we can reformulate [9, Proposition 8] as the following. 
LEMMA 8. The bound

$$
\rho_{1}(x) \ll k^{1 / 2} N^{1 / 4} \log ^{2}(k N) \sigma_{-1}(N) x^{1 / 2}
$$

holds for every $x \geq 1$.

Finally, we need the following estimate.

LEMMA 9. We have

$$
\vartheta_{3}(x) \ll x \log ^{7} x
$$

for every $x \geq 1$.

ProOF. As in [9], we use the Deligne bound

$$
|\lambda(n)| \leq \tau(n) .
$$

Now, by Lemma 6

$$
\begin{aligned}
\vartheta_{3}(x) & =\sum_{n \leq x} \tau(n)^{3} \log ^{2}(x / n) \ll \sum_{1 \leq i \leq \log x+1} i^{2} \sum_{x / e^{i} \leq n \leq x / e^{i-1}} \tau(n)^{3} \\
& \ll \sum_{1 \leq i \leq \log x+1} i^{2} \sum_{n \leq x / e^{i-1}} \tau(n)^{3} \ll x \log ^{7} x \sum_{1 \leq i \leq \log x+1} i^{2} e^{-i} \ll x \log ^{7} x,
\end{aligned}
$$

which finishes the proof.

\section{Proofs}

3.1. Proof of Theorem 1 We note that there is an absolute constant $C_{1}>0$ such that, if we put

$$
X_{1}(k, N)=C_{1} k N \sigma_{-1}(N)^{4} \sigma_{-1 / 2}(N)^{2} \log ^{8}(k N),
$$

then Lemma 7 implies that the bound

$$
\vartheta_{2}(x) \gg \frac{x}{\sigma_{-1}(N) \log (k N)}
$$

holds for $x \geq X_{1}(k, N)$. Using (4) together with Lemma 9 and (2) we see that

$$
\vartheta_{1}(x) \gg \frac{x}{\sigma_{-1}(N)^{2} \log ^{2}(k N) \log ^{7} x}
$$

for $x \geq X_{1}(k, N)$. Let

$$
\begin{gathered}
A_{f}^{+}(x)=\sum_{\substack{n \leq x, \operatorname{gcd}(n, N)=1 \\
\lambda(n)>0}} \lambda(n) \log ^{2}(x / n), \\
A_{f}^{-}(x)=-\sum_{\substack{n \leq x, \operatorname{gcd}(n, N)=1 \\
\lambda(n)<0}} \lambda(n) \log ^{2}(x / n) .
\end{gathered}
$$


Then by Lemma 8,

$$
A_{f}^{+}(x)-A_{f}^{-}(x)=\rho_{1}(x) \ll k^{1 / 2} N^{1 / 4} \log ^{2}(k N) \sigma_{-1}(N) x^{1 / 2} .
$$

From (5), one has

$$
A_{f}^{+}(x)+A_{f}^{-}(x)=\vartheta_{1}(x) \gg \frac{x}{\sigma_{-1}(N)^{2} \log ^{2}(k N) \log ^{7} x} .
$$

We see that (6) and (7) imply that

$$
\min \left\{A_{f}^{+}(x), A_{f}^{-}(x)\right\} \gg \frac{x}{\sigma_{-1}(N)^{2} \log ^{2}(k N) \log ^{7} x}
$$

for $x \geq X_{2}(k, N)$, where

$$
X_{2}(k, N)=C_{2} k N^{1 / 2} \sigma_{-1}(N)^{6} \log ^{22}(k N),
$$

and $C_{2}$ is large enough.

By (3) and the Cauchy inequality

$$
\left(A_{f}^{+}(x)\right)^{2} \leq S_{f}^{+}(x) \sum_{n \leq x} \tau^{2}(n) \log ^{4}(x / n)
$$

Using Lemma 6 and applying the same argument as in Lemma 9, we derive

$$
\sum_{n \leq x} \tau^{2}(n) \log ^{4}(x / n) \ll x \log ^{3} x,
$$

which implies the desired bound for $S_{f}^{+}(x)$. The case of $S_{f}^{-}(x)$ is fully analogous.

3.2. Proof of Theorem 2 Note that, as is well known, $f$ cannot have complex multiplication since by our assumption $N$ is squarefree. Therefore, by [1, Theorem 1], there are some absolute positive constants $\alpha$ and $\beta$ such that, for a sufficiently large real $Z$ and any integer $M \geq 1$ with $M \leq Z^{\beta}$, there exists $s \in\left[Z, Z+Z^{\alpha}\right]$ with $\lambda(s) \neq 0$ and $s \equiv 1(\bmod M)$.

Define

$$
X=\left(x^{\beta} / N\right)^{1 /(4+2 \beta)} .
$$

By Theorem 1 , for $x \geq(k N)^{A}$ with a sufficiently large $A$ (such that $X \geq X(k, N)$ ), there are $m$ and $n$ with $X \leq m<n<X^{2}$ and also with

$$
\operatorname{gcd}(m n, N)=1, \quad \lambda(m) \lambda(n)<0 .
$$

From [1, Theorem 1] we conclude that we can assume that

$$
n \leq m+X^{\gamma} .
$$

For some $\gamma<1$ (provided $x$ is large enough). 
We now put $Z=x / m$ and $M=m n N$. One immediately verifies that $M \leq Z^{\beta}$ for the above choice of $X$. Thus, by [1], we can find $s \in\left[Z, Z+Z^{\alpha}\right]$ with $\lambda(s) \neq 0$ and $s \equiv 1(\bmod M)$. In particular, $\operatorname{since} \operatorname{gcd}(s, n m N)=1$ then, as we have noted before,

$$
\lambda(s m) \lambda(s n)=\lambda(s)^{2} \lambda(m) \lambda(n)<0 .
$$

We also have

$$
\begin{array}{r}
x \leq s m<s n \leq\left(Z+Z^{\alpha}\right)\left(m+X^{\gamma}\right)=x+Z X^{\gamma}+\left(m+X^{\gamma}\right) Z^{\alpha} \\
\leq x+m^{\gamma} Z+2 m Z^{\alpha}
\end{array}
$$

(since $m \geq X$ ) and, after simple calculations, the result follows.

\section{Remarks}

Using the 'individual' bounds

$$
\sigma_{-1}(N) \ll \log \log (N+2), \quad \sigma_{-1 / 2}(N) \ll \exp \left(\frac{\sqrt{\log N}}{\log \log (N+2)}\right),
$$

as well as the bounds 'on average'

$$
\frac{1}{M} \sum_{N \leq M} \sigma_{-1}(N) \ll \frac{1}{M} \sum_{N \leq M} \sigma_{-1 / 2}(N) \ll 1,
$$

which can easily be derived from prime number theory using standard methods of estimating multiplicative functions (see [4, 6]), one can obtain more simplified forms of Theorem 1.

Finally we note that it would be very interesting to obtain an explicit value for the constant $\eta$ in the bound of Theorem 2 .

\section{Acknowledgements}

This idea of this work appeared in October 2006, when the first and third authors attended the conferences 'Number Theory and Beyond' at Kinki University, Osaka, and 'Analytic Number Theory' at the RIMS, Kyoto University. They thank the organizers for their hospitality and the opportunity to participate in these events. The authors are also grateful to Jie $\mathrm{Wu}$ for the careful reading of the manuscript and valuable remarks.

\section{References}

[1] E. Alkan and A. Zaharescu, 'Nonvanishing of Fourier coefficients of newforms in progressions', Acta Arith. 116 (2005), 81-98.

[2] V. Blomer, 'On cusp forms associated with binary theta series', Arch. Math. 82 (2004), 140-146.

[3] Y. J. Choie, W. Kohnen and K. Ono, 'Linear relations between modular forms and non-ordinary primes', Bull. London Math. Soc. 37 (2005), 335-341. 
[4] G. H. Hardy and E. M. Wright, An Introduction to the Theory of Numbers (Oxford University Press, Oxford, 1979).

[5] J. Hoffstein and P. Lockart, 'Coefficients of Maass forms and the Siegel zero', Ann. of Math. 140 (1994), 161-181 (with an appendix by D. Goldfield, J. Hoffstein and D. Lieman).

[6] H. Iwaniec and E. Kowalski, Analytic Number Theory (American Mathematical Society, Providence, RI, 2004).

[7] H. Iwaniec, W. Kohnen and J. Sengupta, 'The first negative Hecke eigenvalue', Int. J. Number Theory 3 (2007), 355-363.

[8] M. Knopp, W. Kohnen and W. Pribitkin, 'On the signs of Fourier coefficients of cusp forms', Ramanujan J. 7 (2003), 269-277.

[9] W. Kohnen and J. Sengupta, 'On the first sign change of Hecke eigenvalues of newforms', Math. Z. 254 (2006), 173-184.

[10] R. Murty, 'Oscillations of Fourier coefficients of modular forms', Math. Ann. 262 (1983), 431-446.

[11] K. Ono, The Web of Modularity: Arithmetic of the Coefficients of Modular Forms and q-series (American Mathematical Society, Providence, RI, 2004).

[12] C. L. Siegel, 'Berechnung von Zetafunktionen an ganzzahligen Stellen', Nachr. Akad. Wiss. Göttingen Math.-Phys. Kl. II (1969), 87-102.

WINFRIED KOHNEN, Mathematisches Institut, Universität Heidelberg, D-69120 Heidelberg, Germany

e-mail: winfried@mathi.uni-heidelberg.de

YUK-KAM LAU, Department of Mathematics, University of Hong Kong, Pokfulam Road, Hong Kong

e-mail: yklau@maths.hku.hk

IGOR E. SHPARLINSKI, Department of Computing, Macquarie University, Sydney, NSW 2109, Australia

e-mail: igor@ics.mq.edu.au 